\section{Review: evidence does not support a reduction in sexual reoffending with psychological interventions, but further high-quality trials are needed}

doi:10.1136/eb-2013-101236

\section{QUESTION}

Question: Do psychological interventions reduce sexual offences in adults who have offended before or are at risk of offending?

Outcomes: Primary outcome: reoffending, as measured by further charge, caution or self-report of sexual offence. Secondary outcomes: cognitive distortions, sexual obsessions or sexually anomalous urges, anxiety, anger, treatment withdrawal and adverse events.

\section{METHODS}

Design: Systematic review and meta-analysis.

Data sources: CENTRAL, MEDLINE, Allied and Complementary Medicine (AMED), Applied Social Sciences Index and Abstracts (ASSIA), Biosis Previews, CINAHL, COPAC, Dissertation Abstracts, EMBASE, International Bibliography of the Social Sciences (IBSS), ISI Proceedings, Science Citation Index Expanded (SCI), Social Sciences Citation Index (SSCI), National Criminal Justice Reference Service Abstracts Database, PsycINFO, OpenSIGLE, Social Care Online, Sociological Abstracts, UK Clinical Research Network Portfolio Database and ZETOC were searched up to October 2010. Authors and experts in the field were contacted for unpublished data and ongoing studies, and reference lists of identified studies were hand searched.

Study selection and analysis: Randomised controlled trials (RCTs) in adults convicted, cautioned or seeking help for sexual offences that compared psychological interventions with each other or controls were included. Meta-analyses were carried out using Review Manager V.5 software. Heterogeneity was assessed using forest plots, $\chi^{2}$ test and $\mathrm{I}^{2}$ statistic. Risk of bias was estimated using the Cochrane Collaboration Tool. Insufficient data were identified for funnel plots, subgroup analysis or sensitivity analysis to be carried out.

\section{MAIN RESULTS}

Ten RCTs met inclusion criteria ( $\mathrm{n}=944$ men, aged 16-72). Sexual offences included paedophilia, rape, exhibitionism and fetishes. Settings included prisons, forensic units, inpatients and outpatients. None of the included studies were blinded and in most cases there was little or no follow-up beyond institutionalisation and into 'at-risk' periods in the community. Five trials of cognitive behavioural therapy (CBT) versus no intervention or standard care were identified but only one trial reported on reoffending. This trial $(\mathrm{n}=484)$ found that 2 years of weekly group and individual CBT had no effect on the rate of reoffending compared with no intervention over 14 years of follow-up (risk ratio $1.10,95 \%$ CI 0.78 to 1.56 ). Four small behavioural intervention studies compared aversion techniques $(n=12)$, imaginal desensitisation ( $\mathrm{n}=20$ and $\mathrm{n}=21$ ), or masturbation prohibition $(n=17)$ versus another behavioural intervention or with wait list control. Reoffending was either not reported for the individual treatment groups or statistical comparisons were not reported. One study $(n=231)$ compared 1 year of group psychodynamic psychotherapy versus probation. A non-significant trend for increased risk of rearrest for sexual offence over 10 years of follow-up was seen for the psychotherapy group (risk ratio $1.87,95 \%$ CI 0.78 to 4.47 ). Limited data were available for secondary outcomes.

\section{CONCLUSIONS}

Currently RCT evidence does not support that psychological interventions reduce the risk of sexual reoffending. High-quality RCTs with minimal bias and long-term follow-up in the community are required to identify interventions that can reduce sexual reoffending rates.

\section{ABSTRACTED FROM}

Dennis JA, Khan 0, Ferriter $\mathrm{M}$, et al. Psychological interventions for adults who have sexually offended or are at risk of offending. Cochrane Database Syst Rev 2012;12: CD007507.

Correspondence to: Conor Duggan, c/o Cochrane Developmental, Psychosocial and Learning Problems Group, Queen's University Belfast, Belfast, UK; conor.duggan@nottingham.ac.uk

Sources of funding: Nottinghamshire Healthcare Trust UK, NHS Cochrane Collaboration Programme Grant Scheme, UK.

- References and Notes section are published online only. To view please visit the journal online (http://dx.doi.org/10.1136/eb-2013-101236).
D ennis and colleagues' recent review is the latest reminder that the research evidence concerning sexual offender treatment is not robust. Previous reviews of quasiexperimental studies have consistently found positive effects of CBT on sexual and general recidivism. All previous reviewers, however, have bemoaned the lack of rigourous studies. Little has changed in the past 15 years. There are still only two large-scale randomised controlled trials (RCTs), with the most recent initiated in the 1980s. Neither found any treatment effects.

The current review is likely to have little impact on practice. Although their search was thorough and their analysis competent, there was not enough evidence to make strong conclusions. Furthermore, the majority of the available RCTs did not examine sexual recidivism, but only intermediate outcomes (such as social anxiety), which may or may not be valid risk indicators.

Sexual offender treatment outcome research appears to be on hold for the time being. There remains, however, an urgent need for more and betterquality research given that many jurisdictions in North America and Europe have enshrined sexual offender treatment in policy. Even the Association for the Treatment of Sexual Abusers believes that high-quality RCTs are needed to advance knowledge and practice. ${ }^{1}$

So what is the evidence-based clinician to do? One option is to consider sexual offenders as, well, offenders. Sexual offending involves both sex crime-specific risk factors (eg, deviant sexual interests) as well as risk factors for general criminality (eg, impulsivity and hostility). Although experts currently debate the extent to which paraphilias are mutable, there is consensus that general criminality declines with age. Furthermore, there is strong evidence that deliberate interventions following the risk, need and responsivity principles ${ }^{2}$ can substantially hasten desistance from crime. Specifically, reductions in criminal recidivism can be expected when the intensity of treatment is commensurate with the offenders' risk for recidivism (risk principle), when treatment targets factors that are empirically related to risk (need principle), and when treatment is delivered in a manner consistent with the offenders' learning style and culture (responsivity principle). These principles seem to apply equally to the treatment of sexual offenders. ${ }^{3}$

\section{R Karl Hanson}

Corrections Research, Public Safety Canada, Ontario, Canada

Competing interests None. 